\title{
Lipopolysaccharides in Food, Food Supplements, and Probiotics: Should We be Worried?
}

\author{
Trudy M. Wassenaar ${ }^{1 *}$ and Kurt Zimmermann ${ }^{2}$ \\ ${ }^{1}$ Molecular Microbiology and Genomics Consultancy, Tannenstrasse 7, 55576 Zotzenheim, Germany \\ ${ }^{2}$ SymbioPharm GmbH, Herborn, Germany
}

Received: 03 July 2018; accepted: 16 July 2018

\begin{abstract}
The fever-inducing effect of lipopolysaccharides (LPS) is well known, and human blood is extremely responsive to this pyrogen. Recently, the safety of LPS-containing food supplements and probiotic drugs as immune-stimulants has been questioned, although these products are orally taken and do not reach the bloodstream undigested. The concerns are understandable, as endotoxaemia is a pathological condition, but the oral uptake of probiotic products containing LPS or Gram-negative bacteria does not pose a health risk, based on the available scientific evidence, as is reviewed here. The available methods developed to detect LPS and other pyrogens are mostly used for quality control of parentally applied therapeuticals. Their outcome varies considerably when applied to food supplements, as demonstrated in a simple comparative experiment. Products containing different Escherichia coli strains can result in vastly different results on their LPS content, depending on the method of testing. This is an inherent complication to pyrogen testing, which hampers the communication that the LPS content of food supplements is not a safety concern.
\end{abstract}

Keywords: LPS, probiotic, safety, oral intake, endotoxaemia, E. coli

\section{Introducing LPS in Health and Disease}

Immunomodulators and food supplements based on bacterial cultures of Gram-negative bacteria, for example containing live Escherichia coli bacteria, are generally well-tolerated products for treatment of human gastrointestinal functional disorders. Due to the nature of these products, they inevitably contain bacterial lipopolysaccharides (LPS). The amount of LPS present depends on the amount and identity of the bacteria or on the treatment that the bacteria have undergone during production.

Bacterial LPS, also known as endotoxin, has been described in association with a number of diseases, including liver damage [1], neurological degeneration (Parkinson's disease and Alzheimer's disease), chronic inflammation of the gut, and diabetes [2]. If an animal is injected with LPS, this will generate a fever, which makes LPS a pyrogenic substance. Nevertheless, the animal gut is the natural environment for large amounts of Gram-negative bacteria that naturally produce LPS, notably members of the phyla Bacteroidetes and, with smaller numbers, Proteobacteria. Thus, a substance that is produced by our natural microflora can cause fever in minute amounts, and is associated with a wide range of diseases, some of which are age-related (neurological degeneration), others life-style or dietary dependent (diabetes and alcoholrelated liver diseases), while yet others may have a genetic component (chronic inflammation). What that means for the safety of probiotics and food supplements that contain a significant amount of LPS is the subject of this contribution.

LPS produces fever via activation of an immunological response involving factors in the blood (complement and Toll-like receptors) that initiate the production of prostaglandins and send signals to the brain to increase body temperature [3]. The link between LPS and neurological diseases is formed by an inflamma-

*Corresponding author: Trudy M. Wassenaar, Molecular Microbiology and Genomics Consultancy, Tannenstrasse 7, 55576 Zotzenheim, Germany; E-mail: trudy@mmgc.eu tory response, which LPS can induce as will be discussed in a following section, while inflammation is also one of the likely triggers for the conditions affecting the brain. However, a direct link between LPS exposure and neurological consequences has only been shown in mice, and even in that model the results are inconsistent [4]. Chronic inflammation is also the key in the link between LPS and diabetes, although in this case the story is complex with many confounding factors [5].

In this contribution, the fate of LPS in a healthy and diseased body is briefly discussed, based on available recent literature studies. In the last part, detection methods of LPS in food supplements are reviewed, and experimental results are exemplary presented.

\section{What is LPS and What Does it Do?}

LPS is a structural component of a bacterial cell; it consists of long chains of sugar moieties (the polysaccharide part) that are covalently connected to lipids. These chains form a dense network that shields the outside of bacteria, forming a gelatinous layer that is attached to the bacterial surface. LPS is the product of enzymes; thus, there are no genes encoding for LPS, but there are genes coding for the enzymes that produce these biomolecules. Its function is to keep the direct outside of bacteria moist and slightly negatively charged, and to shield off compounds that may damage the cell, while the layer is loose enough to let nutrients pass. The real, physical border that separates the inside of a bacterial cell from the outside world is its membrane, a double lipid layer interspersed with proteins, to which LPS is connected via lipid A, a phosphorylated lipid. The toxicity of LPS is mainly due to this lipid A, while the polysaccharides are less toxic. In Gram-negative bacteria, LPS is anchored to the outer membrane via lipid A.

Bacteria release LPS fragments in their environment, while this layer is constantly renewed to maintain its integrity. Humans, like all eukaryotes, have evolved in the presence of bacteria, and

This is an open-access article distributed under the terms of the Creative Commons Attribution-NonCommercial 4.0 International License (https://creativecommons.org/licenses/by-nc/4.0/), which permits unrestricted use, distribution, and reproduction in any medium for non-commercial purposes, provided the original author and source are credited, a link to the CC License is provided, and changes - if any - are indicated. 
since LPS covers the outside of many bacteria, all other living organisms have learned to deal with LPS [6]. In the gut, intestinal phosphatase is produced, which detoxifies lipid A by the removal of phosphate [7]. Some immune cell types of mammals have learned to recognize lipid A and can strongly react to its presence.

Our body is a scaffold for the bacteria that grow on and inside us; bacteria cover all surfaces that are in contact to the outside world. These surfaces are patrolled by immune cells such as monocytes to keep the bacterial population in check, and these cells communicate with each other, with deeper tissue, and with the bacteria that are present; this communication takes place at a molecular level via chemical signals. One of the signals that bacteria produce that is picked up by mammalian cells is LPS. In fact, LPS is a strong immunostimulant, and so is lipid A [6].

\section{LPS is a Strong Immunostimulant}

Although all our outer and inner surfaces are covered by a wide variety of bacteria, deeper tissue should remain free of them. Muscle, fat, nerves, organs, and bones should not contain significant numbers of live bacteria, as their presence could result in severe damage. The body is constantly on the alert to prevent what is typically described as 'infection'. When bacteria reach deeper tissue, which they can do for a variety of reasons, their presence is detected by guarding immune cells that send cytokines as chemical signals to alert other cells, after which recruited killer cells destroy the bacteria and clean up damaged tissue. Monocytes also differentiate into macrophages after they are stimulated by the presence of bacteria. The combined response of producing immune signals, recruiting killer cells, and cleaning up invading bacteria, together with local cell debris, is described as 'inflammation'. The name reminds of the heat that is often generated during this process, which the body employs as it limits bacterial growth and increases effectiveness of the immune attack.

LPS is recognized by immunocytes via Toll-like receptor 4 (TLR4), to which it binds with high affinity. This induces immune cells to produce pro-inflammatory cytokines that trigger inflammation. In particular, immune cells in the blood are highly sensitive to LPS, as the bloodstream would be a perfect mode of transport for bacteria to spread through the body, which the immune system tries to avoid at all costs. When bacteria happen to multiply in the bloodstream (the serious condition of bacterial or infection-induced sepsis), a strong inflammatory response results, and when this response overshoots, it actually causes more damage than the bacteria would do. This is what happens during a septic shock, which can lead to organ failure and death of the patient. One of the triggers that can induce a septic shock is bacterial LPS $[8,9]$.

Inflammation is a complex process with many players. It is tightly regulated so that it can quickly be called upon to raise a strong immune attack when needed, but it also has to shut down in time, for which suppressors are actively produced, and cells are reprogrammed after they performed their necessary tasks [10].

Although LPS is a strong immunostimulant that can induce inflammation, fever, and septic shock, potentially leading to death of the patient, in small doses it can also have positive effects. The immunostimulative effect of LPS has been employed in vaccines, where it was used as an adjuvant. Low levels of endotoxin present in vaccines and other immunotherapeutics lead to TLR activation, with an improved immunization and drug effect as desired outcomes. Acceptable low levels of LPS 'contaminants' with adjuvant activity have been named Innate Immune Response Modulation Impurities (IIRMIs) [11, 12].

\section{Can LPS from Bacteria in the Gut Reach the Bloodstream?}

It has been estimated that the human colon contains approximately 1 -kilogram bacterial mass $\left(10^{12}-10^{13}\right.$ cells). When all these bacteria would produce between 2 and 50 femtograms of LPS, as has been experimentally determined for E. coli [13], this would amount to between 2 and $50 \mathrm{mg}$ LPS in total. Since not all bacteria produce that much LPS, the amount in the gut is likely to be lower. Nevertheless, much higher estimates have been given, of between 10 and 50 grams of endotoxin in a healthy gut [14]. The lethal dose of intravenously injected LPS can be as low as 1 to 2 micrograms [15]. Very low amounts, up to 5 picogram LPS per $\mathrm{mL}$ blood, can be circulating in the bloodstream of a healthy person without side effects [16]. Thus, a normal gut contains between a thousand and a million times more LPS than the lethal dose, an apparent paradox that is explained by compartmentalization - the body will keep LPS inside the gut where it does no harm.

All epithelial surfaces have been selected for during evolution to provide an effective barrier against toxic substances. Gut bacteria and their LPS do not normally damage the epithelial cells lining the gut, as long as these bacteria stay on the lumen side, as demonstrated in early experiments with intestinal segments from rats. Toxicity only arises when LPS reaches the basal side of epithelial cells that normally face deeper tissue [17]. Such a situation can occur when in the gut conditions arise that break the safety barrier, so that bacteria penetrate the mucus, pass through or in between cells, and reach the basal membrane of enterocytes and deeper tissue. Pathogens (for instance, invasive bacteria) frequently employ specific strategies to reach deeper tissue. In the natural course of events, immune cells will detect the invaders and deal with them. In a minority of cases, bacteria may reach the bloodstream, and if they produce high amounts of LPS, septic shock can be the ultimate consequence. A weak immune system increases the chance that this serious complication develops, as a result of bacterial infection, because necessary checks and balances, required to raise an immune response exactly strong enough to resolve the infection, may not function properly. Young infants, in particular neonates whose immune system is not yet fully developed, the very old, and severely ill patients, as well as immunosuppressed individuals, are at higher risk, especially if their skin or mucous surfaces are permanently perforated, for instance by a catheter. Likewise, a damaged, cancerous or inflamed gut may allow bacteria to enter the bloodstream.

Although an intact gut does not allow LPS to pass the mucus and enter deeper tissue, and LPS cannot move by itself, it may hitchhike on transport systems of the gut's endothelial cells; this probably explains why blood LPS levels temporarily rise following a fatty meal, even in healthy individuals [18]. Whether a long-term high-fat diet raises levels of LPS in blood permanently is not consistently shown, with methodological weaknesses possibly being responsible for contradictory results [19]. Any LPS leaving the gut is rapidly redirected to the liver, where it is degraded [20]. When gut lesions are present, it is likely that more LPS reached the bloodstream. Nevertheless, under most conditions, of all LPS produced by bacteria residing in the gut, only a tiny fraction may reach the bloodstream from which it will be rapidly cleared. Only when live bacteria can get into the bloodstream (as some pathogens can do), their LPS can overload the natural protective responses. A sudden exposure to high amounts of LPS is toxic for most cells.

\section{The Fate of LPS that Reaches the Bloodstream}

The fate of LPS or LPS-covered bacteria, once they enter the bloodstream, has been studied in detail in mice. Since the protective response against invasive bacteria is strongly conserved 
between different mammals, the results are by and largely comparable to the human situation, with the exception of patients with severely impaired immune responses.

Free LPS in the blood is rapidly bound to LPS-binding protein, LBP [21]. When unnaturally high doses of LPS were experimentally injected into the colon of mice by means of a rectal enema, it did not result in a local inflammatory reaction, but in the small intestine, indeed inflammation occurred, associated with some tissue damage. After continuing this treatment for five days, the inflammation disappeared, indicating that a sudden increase of LPS in the gut initiates a local but temporary inflammatory reaction [22].

When LPS was directly injected into the bloodstream of mice, it was removed by the liver [23]. By means of fluorescently labelled LPS, it could be demonstrated how the bloodstream was rapidly cleared and how LPS accumulated in the liver where it was broken down [24]. In humans, plasma proteins bind LPS and transfer this to lipoproteins and other acceptors [25].

Although all Gram-negative bacteria produce LPS, not all elicit an equally strong immunological reaction. For instance, a probiotic E. coli strain (DSM 17252) could be injected into the bloodstream of mice without any deleterious effects [26]. These experiments were performed to demonstrate that these E. coli cells preferentially colonize cancer tissue, a characteristic that can actually have diagnostic or therapeutic applications, but the point to note here is that, despite injecting these viable Gram-negative bacteria into the veins of mice, the animals did not suffer from inflammation or septic shock.

\section{Enodotoxaemia and LPS Tolerance}

The term 'endotoxaemia' is used for LPS (endotoxin) originating from the gut that ends up in the blood. As already mentioned, low levels of LPS in blood are perfectly normal. Endotoxaemia is a pathological condition that results from increased intestinal permeability, which can for instance occur during the development of various liver diseases [27]. Alcohol-related liver disease is accompanied with increased LPS levels in the blood [1]. This, and liver conditions not caused by alcohol (e.g., viral hepatitis infections), can result in severe shifts in the gut bacteria, called dysbiosis, with members of the Firmicutes group decreasing and Gram-negative Bacteroidetes increasing; other causes can also result in such a dysbiosis. As particular bacterial species assist in maintaining gut epithelial integrity, a severe intestinal dysbiosis can eventually result in permeability of the gut, with LPS reaching the blood stream, being transported to the liver, and worsening the condition [27]. However, initially, the dysbiosis seems to be a result, not a cause of the liver damage. It should be noted that not the high numbers of intestinal Gram-negative bacteria per se but the increased permeability of the gut is at the basis of endotoxaemia.

Endotoxaemia has also been described in individuals not suffering from liver disease. The condition is now discussed in relation to obesity, diabetes and metabolic syndrome, chronic fatigue, and many other conditions [2]. However, the methods by which LPS is detected in the blood are criticized for being imprecise [28]. Reported LPS levels detected in blood vary considerably between individuals and between methods [29]. It would be difficult to explain how endotoxaemia could be responsible for this wide variety of diseases and conditions, whose incidences have strongly increased over the past decades, while LPS-carrying bacteria have always colonized the intestine. It is quite possible that the bacterial composition in the gut of people living in a modern westernized society has changed, compared to, for example, a century ago.
However, severe changes in the gut microbiome of a large fraction of the population during the past two or three decades are less likely. And even if they had occurred, there must be external factors responsible for these shifts (for instance changes in diet and habits), so that these underlying external factors could just as likely be responsible for the obesity, diabetes, metabolic syndrome, chronic fatigue, and all other conditions and diseases, for which a certain amount of LPS in the blood of these patients is sometimes held responsible [2].

The body can learn from experience, and the adaptive immune system is trained from birth to learn how to respond to stimuli. The learning ability of immune cells means that cells become less sensitive to LPS when they are constantly exposed to low levels. This is called endotoxin or LPS tolerance, a condition that already rises after frequent exposure to low levels of LPS [30]. Endotoxin tolerance results in two effects, of which the first has been known for over 70 years; it results in a form of immunesuppression that protects against cytokine-induced damage [30]. The pyrogenic (fever-inducing) effect of LPS quickly wanes off with repeated administration of LPS, an effect observed in animals and humans alike. The evolutionary importance of LPS tolerance is obvious from the fact that the condition also induces cross-tolerance to bacterial pyrogens other than LPS; this reduces the damage of too strong an immune reaction to other immunogens. LPS-tolerated animals will suffer less from septic shock compared to LPS naïve animals. However, LPS tolerance also results in a second effect that was more recently discovered; it protects animals against systemic infection. When LPS-tolerated animals were challenged with an invasive pathogen such as Salmonella enterica or Staphylococcus aureus, these pathogens were less able to spread and become systemic. Thus, LPS intolerance not only dampens (part of) the immune system to protect against septic shock, but it also supports the immune system, by inhibiting pathogens from spreading [30].

The exact nature of LPS varies between bacterial species and even strains. Although LPS-tolerance is relatively non-specific, variable responses have been demonstrated. This could be expected, since the response of human blood to LPS also varies depending on the bacterial source [28]. LPS produced by Bacteroides dorei (a bacterial species that in the gut has been associated with type I diabetes) differs from LPS produced by E. coli. This difference is important for the onset of autoimmune diseases (to which type I diabetes belongs). Children with relatively high LPS levels from $B$. dorei in their stools during their first three years of life had a higher chance for autoimmune diseases compared to children with high levels of $E$. coli-derived LPS [31]. The authors were able to show that the latter, but not the $B$. dorei-derived LPS, dampened the immune response enough to prevent autoimmune diseases, at least in young children. LPS tolerance could not be induced with 'bad' LPS by $B$. dorei. This implies that exposure at a young age to a certain degree of 'good' LPS in the gut is actually required for the development of a healthy immune system.

\section{Monitoring LPS Concentrations in Pharmaceuticals}

Since LPS concentrations in the bloodstream should be kept to a minimum, it is no surprise that the absence of this and other fever-inducing substances from injected therapeuticals is carefully monitored. According to the European Pharmacopoeia [32], the limit of endotoxin that can be tolerated in parenterally administered substances is 5 endotoxin units (EU) per kg body mass. This specification leads to a maximum tolerable endotoxin amount of $350 \mathrm{EU}$ per individual (based on a body mass of $70 \mathrm{~kg}$ ), or a tolerance of $350 \mathrm{EU} / \mathrm{mL}$ for a parenteral product where a single dose would comprise $1 \mathrm{~mL}$. There 
is no limit available for endotoxin present in orally administered products.

With such strict limits, the quantitative determination of pyrogens, including LPS, in parenterally administered substances is highly important. The first fever-causing agents were identified in 1912 by Hort and Penfold, who were also the first to design a pyrogen test based on injection into rabbits [33]. For decades, this life rabbit test has been the gold standard of pyrogen testing, but the high demand of these tests and ethical considerations about animal use initiated the development of alternative tests, as reviewed elsewhere [34]. The situation improved with the development of the Limulus Amoebocyte Lysate (LAL) test (also known as bacterial endotoxin test [BET]) [35]. This test, which was introduced to clinical medicine in 1970 [36], is based on lysed amoebocytes obtained from the hemolymph of horseshoe crabs. These cells mostly respond to the lipid A moiety of LPS. LAL technology eventually replaced the test using live rabbits. It has been estimated that over $90 \%$ of pyrogenic testing is performed by LAL [37]. Nevertheless, the LAL test has a number of disadvantages: (1) it still requires an animal as the source of reagents, (2) the cells can be stimulated by glucan, a component of the cellulose filters that are used in vaccine production, resulting in false-positive results, and (3) vaccines (the products typically tested for the absence of pyrogenic substances) often contain aluminum hydroxide that interferes with the LAL test, resulting in false-negatives. In addition, a major shortcoming is that the cells only respond to LPS and not to a number of other substances that can also induce fever in humans. This was true for the rabbit blood test (which LAL replaced) as well, as rabbit blood is also less responsive to Gram-positive bacteria than human blood. Obviously, with a test that does not detect all pyrogens, fever-inducing contaminations may remain undetected.

Within the last decades, animal welfare became a serious issue, and the replacement of animal tests with non-animal technology became a target. Eventually, a test based on human whole blood was developed as an alternative to the LAL test, which became known as Monocyte Activation Test (MAT). Since MAT uses human blood cells, it has the advantage that it responds to all pyrogens that potentially induce fever in humans [38]. With the use of frozen blood cells (cryo-based blood assays), accessibility to freshly drawn human blood was no longer needed, and individual variation was minimized [39]. After validation, MAT was included as a standard method for pyrogen detection in the European Pharmacopoeia in 2010 [32]. The EU directive 2010/63/EU [40] required a change in the German animal welfare law, and this resulted in implementation of new national regulation guidelines for the use of experimental animals [41, 42].

Developments did not stop there, as the production of recombinant horseshoe crab clotting factor $\mathrm{C}(\mathrm{rFC})$ [43] allowed novel in vitro ELISA tests to become available. However, these tests do not overcome the limitation that horse shoe crab enzymes do not respond to all pyrogenic substances in the same manner as human blood does, and currently, recombinant-protein based testing is only performed in a minority of routine testing laboratories.

\section{Is it Safe to Ingest LPS?}

Most pyrogenic testing is performed to avoid contamination of products that will be administered by injection, but there are no safety limits for LPS in foodstuffs or food supplements, as oral intake does not result in fever or other known side effects.

Few people would voluntarily swallow purified LPS, but some probiotic products contain large numbers of viable Gram-negative E. coli bacteria, and these products inevitably contain LPS. The quantitative effect of this extra LPS in the gut is negligible. According to the most accurate estimate, the human gut of an 'average' $70 \mathrm{~kg}$ reference person contains approximately $3.8 \times 10^{13}$ bacterial cells [44]. Proteobacteria, to which E. coli belong, make up about $4.5 \%$ of these, though this fraction can increase to $15 \%$ in an inflamed gut during dysbiosis [45]. Even during that condition, these numbers are still low compared to the other Gram-negative phylum that is more abundantly present in the gut, the Bacteroidetes; these typically make up $65 \%$ of a healthy microbiome [45]. These two LPS-producing phyla together would represent approximately $2.6 \times 10^{13}$ cells. Obviously, a regular intake of a probiotic based on E. coli, such as Mutaflor ${ }^{\circledR}$ or Symbioflor $2^{\circledR}$, with a typical daily dose of $10^{7}$ to $10^{9}$ cells, would not make any difference to these numbers, or to the amount of LPS they produce. There is a negligible risk for such products to raise the chance of endotoxaemia or to result in other negative side effects.

\section{Difficulties with Quantitative LPS Determination in Bacterial Food Supplements}

The performance of currently available LPS quantitative tests was compared in a small study of 6 commercial products (probiotics or autovaccines that are sold for their immunomodulant activities) that contain bacteria or bacterial components and could be expected to contain LPS.

In a first experiment, performed by an accredited laboratory, the classical LAL test was performed as described in Chapter 2.6.14 ("Bacterial Endodoxins") and the MAT as in Chapter 2.6.30 ("Monocyte-activation test") of the European Pharmacopoeia [32]. The latter test was performed using cryo-preserved peripheral blood mononuclear cells in combination with ELISA detection of IL- 6 . The sensitivity of the MAT method turned out to be approximately 0.1 endotoxin equivalent units per $\mathrm{mL}$. The third method used was performed with a commercial test product that is based on recombinant horseshoe crab clotting factor $\mathrm{C}$ (rFC). The test has a sensitivity of 0.01 endotoxin units (EU) per $\mathrm{mL}$, as declared by the vendor; in Chapter 5.1 .10 of the European Pharmacopoeia ("Guidelines for using the test for bacterial endotoxins") [32], a sensitivity of $0.05 \mathrm{EU} / \mathrm{mL}$ is given. The results of this pilot study are summarized in Table 1.

Product 1 is a probiotic product containing a suspension of viable Gram-positive bacteria (Enterococcus faecalis); two production batches were compared, which produced similar results. Product 2 is a probiotic that contains viable $E$. coli bacteria, which explains the high amount of endotoxin that was determined. Two batches were analyzed that differed by a factor of 1.8 (as per LAL), $\geq 0.65$ (as per MAT) or 1.5 (as per rFC test). Inter-test comparisons produced higher findings by MAT than by LAL or rFC. Product 3 is an immunostimulant and contains a mixture of the heat-inactivated bacteria of products 1 and 2 . The amount of endotoxin measured here was 10 to 20 times lower than that in product 2, with higher amounts in batch 1 compared to batch 2 , for all three methods. However, the $\mathrm{rFC}$ test turned out to be insensitive to the endotoxin of product 3, resulting in more than 100-times lower values compared to MAT. Again, MAT recorded the highest values for endotoxin units, which is consistent with the findings for product 2, as the main LPS source in product 3 is the same E. coli strain as that in product 2. Product 4 is also a probiotic containing inactivated $E$. coli, but a different strain to that of products 2 and 3 . The results suggest that the inactivation completely destroyed all LPS, as the final product did not contain endotoxin above the detection levels of the tests. Product 5, on the other hand, contained very high amounts of endotoxin, which was to be expected, as this is a probiotic 
Table 1. Results of three quantitative endotoxin tests by laboratory 1

\begin{tabular}{lcccc}
\hline Products for oral use & Content & $\begin{array}{c}\text { Endotoxin by LAL } \\
(\mathrm{EU} / \mathrm{mL})\end{array}$ & $\begin{array}{c}\text { Endotoxin by MAT } \\
(\mathrm{EEU} / \mathrm{mL})^{a}\end{array}$ & $\begin{array}{c}\text { Endotoxin by } \mathrm{rFC} \\
(\mathrm{EU} / \mathrm{mL})\end{array}$ \\
\hline Product 1, batch 1 & Viable Ent. faecalis DSM 16440 & $<200$ & $<100$ & $<100$ \\
Product 1, batch 2 & & $<200$ & $<100$ & $<100$ \\
Product 2, batch 1 & Viable E. coli DSM 17252 & 1750 & $>4000$ & 1371 \\
Product 2, batch 2 & & 928 & $>4000$ & 903 \\
Product 3, batch 1 & Lysed Ent. faecalis DSM 16440 & 51 & $147.8 \pm 426.2$ & 1.2 \\
Product 3, batch 2 & plus E. coli DSM 17252 & 33 & $105.1 \pm 4.9$ & 0.9 \\
Product 3, batch 3 & & n.d. & $<1.7 \pm 6.6$ & n.d. \\
Product 4 & Lysed E. coli Laves & $<1.00^{b}$ & $<0.5^{b}$ \\
Product 5 & Viable E. coli DSM 6601 & 397,000 & $<1.00^{b}$ & $<3,400$ \\
Product 6 & E. coli extract ATCC 25922 & $<100,000$ & 100,000 & $<5,000$ \\
Product 7 & E. coli extract ENR 2176116 & $<100,000$ & 165 & $<5,000$
\end{tabular}

${ }^{a}$ EEU: endotoxin equivalent units. The samples were diluted up to 4000 times in endotoxin-free water prior to testing with a dilution factor determined by pilot experiments.

${ }^{b}$ Below the detection limit of the method.

with yet another strain of viable $E$. coli cells. Again, the rFC test performed rather poorly, as it detected 5.4-times lower endotoxin levels than LAL. Note that this was not the case with Product 2. The different E. coli strains present in products 2 and 5 apparently affected the performance of the different tests. It can be concluded that LAL can produce higher scores than MAT with one strain, but lower scores with another strain of $E$. coli. Lastly, two extracts of different $E$. coli strains were tested in products 6 and 7 that are administered as nasal spray as immunostimulants (autovaccines). Surprisingly, the endotoxin of these strains could hardly be detected by MAT; this suggests that the endotoxin present in these extracts would not be pyrogenic to humans. Nevertheless, much higher scores were obtained with LAL and $\mathrm{rFC}$, whereby the endotoxin levels detected by LAL in these extracts were 20 times higher than the rFC findings.

The range of the resulting outcomes is rather extreme, and the precision of the reported findings widely varies as well. Some results are highly precise $(105.1 \pm 4.9 \mathrm{EEU} / \mathrm{mL}$ for MAT, product 3, batch 2) but other samples resulted in an inacceptable error rate with the same method $(147.8 \pm 426.2 \mathrm{EEU} / \mathrm{mL}$ for MAT, same product 3, batch 1). Other reported results are highly imprecise and at most provide a rough estimate on the endotoxin content only ( $>4000$ or $<200)$. Some results are even close to meaningless, like $<5000$ (which can be anything from zero to $4999 \mathrm{EU} / \mathrm{mL}$ ) or even $<100,000$. Normally, such results would be inacceptable in a scientific publication, but they are included here because this is a typical output from an accredited laboratory, using standardized and internationally accepted methods.

A selection of the products was also analyzed by a second, independent, accredited laboratory. This laboratory reported the results for three different dilutions and kindly calculated the mean values of these measurements, although they were not true replicates. Whether replicates had been performed, as the EU standard dictates [46], was not clear. Only when a test is repeated as independent, truly replicate experiments, calculation of a mean and standard deviation would be acceptable.
The results obtained from this second analysis are summarized in Table 2. The reported results are corrected for the stated dilution factor.

Again, there is variation in the results, but within one method and product, the variation between the different dilutions is not as extensive as those reported by the first laboratory. In general, MAT results report much higher values than either LAL or the rFC test. The expected highest endotoxin content of product 2 was confirmed in all three tests, and a lower content in product 1 (Gram-positive bacteria only) compared to product 3 (Gram-positive plus Gram-negative bacteria) was also confirmed, but only by LAL. Between the three different test assays, a three-fold difference was found for product 2 (highest values were reported by MAT, lowest by LAL). For product 1, MAT reported 10,000 times higher concentrations than LAL, while the results from $\mathrm{rFC}$ were inconclusive. For product 3, there were few finite results to be compared, but LAL apparently detected 2.5 times more endotoxin than MAT did, for the same dilution. Thus, depending on the product, either MAT (product 2) or LAL (product 3) detected more endotoxin.

A comparison between the results of the two laboratories for product 2 is acceptable. The $>4000 \mathrm{EEU} / \mathrm{mL}$ endotoxin present in product 2 , determined by MAT (lab 1 ) roughly corresponded with $3800-5800 \mathrm{EU} / \mathrm{mL}$ determined in lab 2 with the same method, acknowledging that the analysed production batches of the product had not been identical. For this product 2, the order of magnitude of endotoxin detected by the rFC tests was also similar between both labs (903$1371 \mathrm{EU}$ and 1010-1570 EU for lab 1 and 2, respectively), as were LAL results (928-1750 EU and 499-746 EU for lab 1 and 2, respectively). An inter-laboratory comparison for the other two products is more problematic. The endotoxin results for product 1 as determined by LAL $(<200 \mathrm{EU})$ by lab 1 are strictly speaking not conflicting the results from lab 2 , but with their value between 0.01 and $0.08 \mathrm{EU} / \mathrm{mL}$, it is highly questionable if the output of lab 1 was meaningful. The same is true for the output of $<100 \mathrm{EU} / \mathrm{mL}$ endotoxin as determined by $\mathrm{rFC}$ for product 1 , which by lab 2 was reported as

Table 2. Results of three quantitative endotoxin tests by laboratory 2

\begin{tabular}{|c|c|c|c|c|}
\hline Products for oral use & Content & $\begin{array}{l}\text { Endotoxin by LAL } \\
(\mathrm{EU} / \mathrm{mL})\end{array}$ & $\begin{array}{c}\text { Endotoxin by MAT } \\
(\mathrm{EU} / \mathrm{mL})\end{array}$ & $\begin{array}{c}\text { Endotoxin by rFC } \\
(\mathrm{EU} / \mathrm{mL})\end{array}$ \\
\hline \multirow[t]{2}{*}{ Product 1 , batch 3} & Viable Ent. faecalis DSM 16440 & $1: 1,0.0659$ & $1: 200,154.4$ & $1: 10,<0.05$ \\
\hline & & $1: 2,0.0846$ & $1: 500,78.5$ & $1: 100,<0.50$ \\
\hline \multirow[t]{3}{*}{ Product 2, batch 3} & Viable E. coli DSM 17252 & $1: 500,499$ & $1: 7000,3829$ & $1: 500,1570$ \\
\hline & & $1: 1000,500$ & $1: 8000,5072$ & $1: 1000,1360$ \\
\hline & & $1: 10000,746$ & $1: 10000,5800$ & $1: 10000,1010$ \\
\hline \multirow[t]{2}{*}{ Product 3 , batch 4} & Lysed Ent. faecalis DSM 16440 plus & $1: 10,2.72$ & $1: 20,<2.50$ & $1: 10,<0.05$ \\
\hline & & $1: 100,7.44$ & $1: 200,<25$ & $1: 100,<0.50$ \\
\hline
\end{tabular}


$<0.05$ or $<2.50 \mathrm{EU} / \mathrm{mL}$. For product 3 , the findings by MAT could not be compared due to their high spread (between 61147 by lab 1, with an unacceptable error, and between $<2.5$ and 6.40 by lab 2).

Overall, these findings illustrate the difficulty in determining endotoxin in a given product, even if the product contains E. coli bacteria or components thereof. The findings with one test can vastly differ with results obtained by another method, depend on the $E$. coli strain being present, and also vary considerably between laboratories. That different bacterial species produce different types of LPS being able to evoke stronger or weaker human blood responses has been pointed out before [29]. Here, we observe that even for different strains of the same species (E. coli), there is no guarantee that their LPS is detected at equal levels in every test.

How can these rather imprecise results be explained, in view of the high accuracy, sensitivity, and reproducibility that are required for pyrogen testing in vaccine production? The limitations of LAL and other tests derived from horseshoe crab proteins were already discussed; their results do not always reflect the true pyrogenic potential of compounds to human blood. Both over- and under-reactivity can be obtained. In addition, these tests were developed and validated to determine very low amounts of endotoxin. Food supplements based on Gram-negative bacteria contain such vast amounts of LPS that they must be strongly diluted prior to analysis. This introduces inaccuracies that are not encountered in the tests performed for quality controlling parenteral products.

The outcome of this small comparison further demonstrates the struggle of producers of probiotics who aim for clear statements on the contents of their products, but who depend on external accredited laboratories for analyses, which do not report findings with sufficient reproducibility and do not clearly state the results of replicate analyses as a standard service.

\section{Conclusions}

When patients are diagnosed with endotoxaemia, where LPS is detected in unacceptable amounts in their blood, this should be taken seriously, as this may be an indication for liver damage. However, the deliberate intake of Gram-negative bacteria is not harmful as a result of bacterial LPS production, provided they are not pathogenic. LPS-producing bacteria are normal components of the intestinal microbiome of a healthy gut. Only in severe cases, where dysbiosis has severely distorted the ratio between Gram-positive and Gram-negative bacteria in the gut, may the latter be a source for clinical endotoxaemia, and further diagnosis would be required to identify the underlying cause. A dysbiosis cannot be induced by intake of products containing viable Gramnegative bacteria, as their numbers are too low to influence the overall ratio of Gram-negative and Gram-positive bacteria in the gut. Such products will not elevate LPS levels in the blood. A large meal high in fat can be expected to have a stronger endotoxemic effect than a dose of an E. coli-containing probiotic.

Some of the literature studies cited here is decades old, but that doesn't make the observations less valid. It has been known for nearly a century that LPS should not exceed low but critical levels in the bloodstream and that endotoxin has a high toxicity when injected. But that toxicity is not related to oral uptake, and this must be clearly communicated to a general public, so that unnecessary scares are avoided. There is no safe oral dose of LPS, for the simple reason that LPS is not toxic when ingested.

\section{Funding Sources}

This review was financially supported by SymbioPharm $\mathrm{GmbH}$; the company had no influence on the content of the work.

\section{Author Contributions}

Both authors have equally contributed to the work.

\section{Conflict of Interest}

T.M.W. works as a consultant for companies producing probiotics, including products that contain E. coli. K.Z. works for SymbioPharm GmbH and contributed to this work on personal title.

\section{References}

1. Magdaleno F, Blajszczak CC, Nieto N. Key events participating in the pathogenesis of alcoholic liver disease. Biomolecules. 2017;7:pii:E9.

2. Gomes JM, Costa JA, Alfenas RC. Metabolic endotoxemia and diabetes mellitus: A systematic review. Metabolism. 2017;68:133-44.

3. Roth J, Blatteis CM. Mechanisms of fever production and lysis: lessons from experimental LPS fever. Compr Physiol. 2014;4:1563-604.

4. Catorce MN, Gevorkian G. LPS-induced murine neuroinflammation model: main features and suitability for pre-clinical assessment of nutraceuticals. Curr Neuropharmacol. 2016;14:155-64.

5. Laugerette F, Vors C, Peretti N, Michalski MC. Complex links between dietary lipids, endogenous endotoxins and metabolic inflammation. Biochimie. 2011;93:39-45.

6. Neyen C, Lemaitre B. Sensing Gram-negative bacteria: a phylogenetic perspective. Curr Opin Immunol. 2016;38:8-17.

7. Beumer C, Wulferink M, Raaben W, Fiechter D, Brands R, Seinen W. Calf intestinal alkaline phosphatase, a novel therapeutic drug for lipopolysaccharide (LPS)-mediated diseases, attenuates LPS toxicity in mice and piglets. J Pharmacol Exp Ther. 2003;307:737-44.

8. Opal SM. Endotoxins and other sepsis triggers. Contrib Nephrol. 2010;167:14-24

9. Salomao R, Brunialti MK, Rapozo MM, Baggio-Zappia GL, Galanos C, Freudenberg M. Bacterial sensing, cell signaling, and modulation of the immune response during sepsis. Shock. 2012;38:227-421.

10. Netea MG, Balkwill F, Chonchol M, Cominelli F, Donath MY, Giamarellos-Bourboulis EJ, et al. A guiding map for inflammation. Nat Immunol. 2017;18:826-31.

11. Verthelyi D, Wang V. Trace levels of innate immune response modulating impurities (IIRMIs) synergize to break tolerance to therapeutic proteins. PLoS One. 2010;5:e15252

12. Haile LA, Puig M, Kelley-Baker L, Verthelyi D. Detection of innate immune response modulating impurities in therapeutic proteins. PLoS One. 2015; 10:e0125078.

13. Watson SW, Novitsky TJ, Quinby HL, Valois FW. Determination of bacterial number and biomass in the marine environment. Appl Environ Microbiol. 1977;33:940-6.

14. Bonenberger J, Diekmann W, Fennrich S, Fischer M, Friedrich A, Hansper M, et al. Pyrogentestung im Vollblut. Bundesgesundheitsbl Gesundheitsforsch Gesundheitsschutz. 2000;43:525-33.

15. Sauter C, Wolfensberger C. Interferon in human serum after injection of endotoxin. Lancet. 1980;2:852-3.

16. Salden HJ, Bas BM. Endotoxin binding to platelets in blood from patients with a sepsis syndrome. Clin Chem. 1994;40:1575-9.

17. Osman NE, Weström B, Karlsson B. Serosal but not mucosal endotoxin exposure increases intestinal permeability in vitro in the rat. Scand J Gastroenterol. 1998;33:1170-4.

18. Erridge C, Attina T, Spickett CM, Webb DJ. A high-fat meal induces lowgrade endotoxemia: evidence of a novel mechanism of postprandial inflammation. Am J Clin Nutr. 2007;86:1286-92.

19. Boutagy NE, McMillan RP, Frisard MI, Hulver MW. Metabolic endotoxemia with obesity: Is it real and is it relevant? Biochimie. 2016;124:11-20.

20. Guerville M, Boudry G. Gastrointestinal and hepatic mechanisms limiting entry and dissemination of lipopolysaccharide into the systemic circulation. Am J Physiol Gastrointest Liver Physiol. 2016;311:G1-15.

21. Tobias PS, Soldau K, Ulevitch RJ. Identification of a lipid A binding site in the acute phase reactant lipopolysaccharide binding protein. J Biol Chem. 1989;264:10867-71.

22. Im E, Riegler FM, Pothoulakis C, Rhee SH. Elevated lipopolysaccharide in the colon evokes intestinal inflammation, aggravated in immune modulatorimpaired mice. Am J Physiol Gastrointest Liver Physiol. 2012;303:G490-7.

23. Yao Z, Mates JM, Cheplowitz AM, Hammer LP, Maiseyeu A, Phillips GS, et al. Blood-borne lipopolysaccharide is rapidly eliminated by liver sinusoidal endothelial cells via high-density lipoprotein. J Immunol. 2016;197:2390-9.

24. Duheron V, Moreau M, Collin B, Sali W, Bernhard C, Goze C, et al. Dual labeling of lipopolysaccharides for SPECT-CT imaging and fluorescence microscopy. ACS Chem Biol. 2014;9:656-62.

25. Vesy CJ, Kitchens RL, Wolfbauer G, Albers JJ, Munford RS. Lipopolysaccharide-binding protein and phospholipid transfer protein release lipopolysaccharides from Gram-negative bacterial membranes. Infect Immun. 2000;68:2410-7.

26. Kocijancic D, Felgner S, Frahm M, Komoll RM, Iljazovic A, Pawar V, et al. Therapy of solid tumors using probiotic Symbioflor-2: restraints and potential. Oncotarget. 2016;7:22605-22.

27. Acharya C, Bajaj JS. Gut microbiota and complications of liver disease. Gastroenterol Clin North Am. 2017;46:155-69.

28. Munford RS. Endotoxemia-menace, marker, or mistake? J Leukoc Biol. 2016;100:687-98. 
29. Gnauck A, Lentle RG, Kruger MC. Chasing a ghost? Issues with the determination of circulating levels of endotoxin in human blood. Crit Rev Clin Lab Sci. 2016;53:197-215.

30. Seeley JJ, Ghosh S. Molecular mechanisms of innate memory and tolerance to LPS. J Leukoc Biol. 2017;101:107-19.

31. Vatanen T, Kostic AD, d'Hennezel E, Siljander H, Franzosa EA, Yassour M, et al. Variation in Microbiome LPS Immunogenicity Contributes to Autoimmunity in Humans. Cell 2016;165:842-53. Erratum in: Cell 2016; $165: 1551$.

32. European Pharmacopoeia, $9^{\text {th }}$ edition. 2017. Available from: https:// www.edqm.eu/en/european-pharmacopoeia-9th-edition.

33. Hort EC, Penfold WJ. Microorganisms and their relation to fever. J Hyg (Lond). 1912;12:361-90.

34. Fennrich S, Hennig U, Toliashvili L, Schlensak C, Wendel HP, Stoppelkamp S. More than 70 years of pyrogen detection: Current state and future perspectives. Altern Lab Anim. 2016;44:239-53.

35. Rojas-Corona RR, Skarnes R, Tamakuma S, Fine J. The Limulus coagulation test for endotoxin. A comparison with other assay methods. Proc Soc Exp Biol Med. 1969;132:599-601.

36. Levin J, Poore TE, Zauber NP, Oser RS. Detection of endotoxin in the blood of patients with sepsis due to gram-negative bacteria. N Engl J Med. 1970;283:1313-6.

37. Hartung T. The human whole blood pyrogen test - lessons learned in twenty years. Altex. 2015;32:72-100.
38. Hartung T, Wendel A. Die Erfassung von Pyrogenen in einem humanen Vollblutmodell. Altex. 1995; 12:70-5.

39. Schindler S, Asmus S, von Aulock S, Wendel A, Hartung T, Fennrich S. Cryopreservation of human whole blood for pyrogenicity testing. J Immunol Methods. 2004;294:89-100

40. Directive 2010/63/EU of the European Parliament and of the Council of 22 September 2010 on the protection of animals used for scientific purposes. Available from: http://data.europa.eu/eli/dir/2010/63/oj. Last accessed on 2 July 2018

41. Bundesministerium der Justiz und für Verbraucherschutz, Verordnung zum Schutz von zu Versuchszwecken oder zu anderen wissenschaftlichen Zwecken verwendeten Tieren (Tierschutz-Versuchstierverordnung - TierSchVersV), 31.8.2015. Available from: https://www.gesetze-im-internet.de/tierschversv/BJNR312600013. html. Last accessed on 2 July 2018.

42. Bundesministerium der Justiz und für Verbraucherschutz, Tierschutzgesetz, 29.07.2017. Available from: https://www.gesetze-im-internet.de/tierschg/TierSchG pdf. Last accessed on 2 July 2018.

43. Ding JL, Ho B. Endotoxin detection from limulus amebocyte lysate to recombinant factor C. Subcell Biochem. 2010;53:187-208.

44. Sender R, Fuchs S, Milo R. Revised estimates for the number of human and bacteria cells in the body. PLoS Biol. 2016;14:e1002533.

45. Shin NR, Whon TW, Bae JW. Proteobacteria: microbial signature of dysbiosis in gut microbiota. Trends Biotechnol. 2015;33:496-503.

46. Available from: http://www.ema.europa.eu/docs/en_GB/document_library/ Scientific_guideline/2010/09/WC500097056.pdf. Last accessed on 2 July 2018. 